\section{Infecções dos espaços cervicais: estudo prospectivo de 57 casos}

\author{
Luiz Ubirajara Sennes ${ }^{1}$, Rui Imamura ${ }^{2}$, Fernando \\ Veiga Angélico Júnior ${ }^{3}$, Lucinda Simoceli ${ }^{4}$, Ronaldo \\ Frizzarini $^{4}$, Domingos Hiroshi Tsuji ${ }^{2}$
}

Resumo

$\mathbf{O}$ bjetivo: apesar da antibioticoterapia, as infecções dos espaços cervicais profundos continuam a ter grande importância, tanto por sua alta incidência, como pelo risco de complicações. Forma de estudo: clínico, prospectivo. Material e método: descrevem-se neste estudo 57 casos de pacientes com diagnóstico de infecção cervical (celulite, abscesso e fasceíte necrotizante) internados na Enfermaria de Otorrinolaringologia do Hospital das Clínicas da Faculdade de Medicina da USP entre janeiro de 1999 e janeiro de 2001. Cinqüenta pacientes $(87,7 \%)$ apresentavam abscesso cervical, cinco $(8,8 \%)$, celulite e dois pacientes $(3,5 \%)$, fasceíte necrotizante. A infecção odontogênica foi o foco de origem mais prevalente, responsável por 24 casos (42\%), seguida das amigdalites $(17,5 \%)$ e linfadenites $(15,8 \%)$. Os espaços cervicais mais acometidos foram o submandibular $(57,9 \%)$, o sublingual $(26,3 \%)$ e o parafaríngeo $(24,6 \%)$. Em 53 pacientes realizaramse culturas das quais $71,7 \%$ foram positivas, sendo que $20,8 \%$ destas apresentaram flora mista. Os microorganismos mais prevalentes dentre os aeróbicos foi o Streptococcus viridans $(41,5 \%)$ e, dentre os anaeróbios, Peptostreptococcus micros e Peptostreptococcus sp $(5,7 \%$ e $3,8 \%$, respectivamente). A associação entre penicilina cristalina e metronidazol foi o tratamento preconizado em 31 pacientes $(54,3 \%)$. Nos demais, diversos antibióticos como clindamicina, cefalosporinas de terceira geração, cloranfenicol e oxacilina foram utilizados. Cinqüenta e quatro pacientes $(94,7 \%)$ evoluíram satisfatoriamente, dois $(3,5 \%)$ apresentaram deiscência da ferida cirúrgica e um $(1,7 \%)$ foi a óbito por mediastinite. Conclusão: os abscessos cervicais ainda configuram uma importante causa de morbidade em nossa população e suas complicações requerem vigilância para a prevenção e abordagem oportunas, especialmente pelo tratamento mais adequado.

\section{Deep neck infections: prospective study of 57 patients}

Palavras-chave: abscessos cervicais, fasceíte necrotizante, espaços cervicais.

Key words: deep neck space, necrotizing fasciitis, neck space infections.

\section{Summary}

\begin{abstract}
A Since the development of penicillin, the head and neck space infections have become relatively uncommon, although the morbidity remains high. The delayed diagnosis and treatment can cause serious complications as airway obstruction, mediastinitis, sepsis and death. Controversy still exists about choice of antibiotic therapy, diagnosis, and clinical or surgical treatment. The aim of this prospective study is analyze the etiology (associated factors, site of origin and bacteriology), clinical presentation (symptoms, origin, primary and secondary involved spaces and the routes of propagation of the infection), treatment (antimicrobial therapy, corticosteroid, surgical drainage) and complications. Study design: Prospective clinical. Material and method: This prospective series included 58 patients that need hospitalization due to deep neck infection in a two-year period, from December 1997 to December 1999. In all cases there was confirmation of the diagnosis by cervical ultrasound and/or CT scanning. The management consisted of maintenance of an adequate airway and systemic antibiotics. Surgical drainage was performed promptly as required. Bacteriological cultures were done when purulent material was found in spontaneous drainage and during surgical procedures. Results: Thirty patients were males and 28 females. The mean age was 33,4 years. The most common site of infection was the submandibular (46,6\%), followed by pterygomandibular (31\%), sublingual (29,3\%) and parapharyngeal space (20,7\%). Thirty-two patients had infection in more than one site. The etiology was odontogenic (40\%), suppurative adenitis (13,8\%), salivary gland infection (10,3\%). Surgical drainage was performed in $72,4 \%$ of patients. The association of penicillin and metronidazole was used in $55.2 \%$ of cases and clindamicin in $13.8 \%$. The cultures were negative in $38.1 \%$. S. viridans, S. aureus, S. pyogenes, H. influenzae and Peptostreptococcus were isolated. Recovery occurred in 57 patients. One patient died due to mediastinal spread of infection. Two patients had dehiscence of the suture and one had infection recurrence. Conclusion: Deep neck space infections are potential severe disease and a challenging problem in the care of children and adults. An accurate diagnosis based in image studies is related with less complications and sequels. Odontogenic infections are the most prevalent etiology. The association of penicillin and metronidazole still is a cheap and effective treatment to the most cases. The early and wide drainage is the key to infection control, and is related to quickly recuperation and hospital discharge.
\end{abstract}

\footnotetext{
${ }^{1}$ Professor Livre Docente da Disciplina de Otorrinolaringologia da Faculdade de Medicina da Universidade de São Paulo. Assistente-Doutor da Divisão de Clínica Otorrinolaringológica do Hospital das Clínicas da Faculdade de Medicina da Universidade de São Paulo. ${ }^{3}$ Pós-graduando da Disciplina de Otorrinolaringologia da Faculdade de Medicina da Universidade de São Paulo. ${ }^{4}$ Residente da Disciplina de Otorrinolaringologia da Faculdade de Medicina da Universidade de São Paulo

Trabalho realizado na Divisão de Clínica Otorrinolaringológica do Hospital das Clínicas da Faculdade de Medicina da Universidade de São Paulo - Serviço do Prof. Aroldo Miniti Trabalho Apresentado no II Congresso Triológico de Otorrinolaringologia, Goiânia, 22 a 26 de agosto de 2001 Categoria: Tema Livre - Selecionado para premiação, recebendo Menção Honrosa.

Endereço para correspondência: Lucinda Simoceli - Rua Mourato Coelho, 756 Ap: 65 - Pinheiros - São Paulo - 05417-001 Tel. (0xx11) 9383.6673 Fax: (0xx11) 3280.0299 - E-mail: 1simoceli@bol.com.br Artigo recebido em 00/00/00. Artigo aceito em 00/00/00.
} 


\section{INTRODUÇÃO}

Desde 1836, quando Wilhelm Friedrich von Ludwig ${ }^{1}$ descreveu a chamada Angina de Ludwig, várias discussões ocorreram sobre as diversas manifestações clínicas e tratamento das infecções e abscessos profundos do pescoço.

A evolução dos métodos diagnósticos, o descobrimento de novos antibióticos e a abordagem cirúrgica mais precisa e agressiva, aliados à prevenção das infecções em cabeça e pescoço proporcionaram o aumento da sobrevida dos pacientes e a queda da incidência de complicações maiores.

Devido às características anatômicas dos espaços cervicais, apesar do uso de antibióticos adequados, as infecções nos espaços cervicais profundos se disseminam através das fáscias cervicais, originam abscessos e envolvem estruturas vitais como as vias aéreas superiores, vísceras cervicais e órgãos torácicos.

Além das manifestações locais, que resultam do efeito compressivo do abscesso ou da inflamação, levando ao desenvolvimento de edema, espasmos musculares irritativos

Gráfico 1. Distribuição da faixa etária dos pacientes

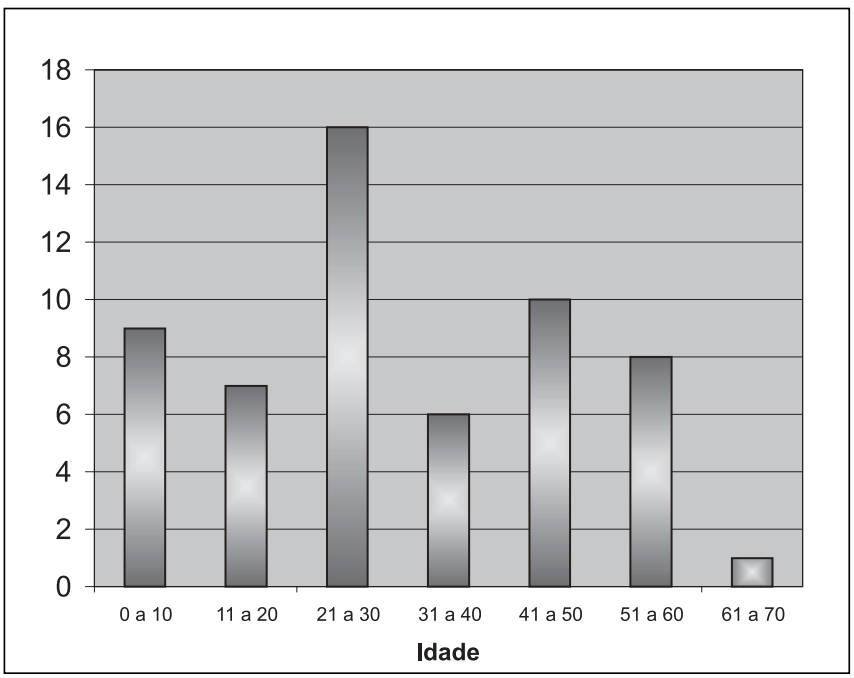

Tabela 1. Distribuição das etiologias das infecções cervicais profundas

\begin{tabular}{lc}
\hline Etiologia & $\mathrm{N}(\%)$ \\
\hline Infecção odontogênica & $24(42,1 \%)$ \\
Amigdalite & $10(17,5 \%)$ \\
Linfadenite pós IVAS & $9(15,8 \%)$ \\
Causa Indefinida & $5(8,8 \%)$ \\
Infecções de pele & $3(5,3 \%)$ \\
Ingestão de Corpo Estranho & $2(3,5 \%)$ \\
Submandibulite & $1(1,7 \%)$ \\
Cisto Branquial Infectado & $1(1,7 \%)$ \\
Parotidite & $1(1,7 \%)$ \\
Necrose Tumoral & $1(1,7 \%)$ \\
\hline Total & $57(100 \%)$ \\
\hline
\end{tabular}

e obstrução do trato aerodigestivo, também apresentam alterações sistêmicas como febre, letargia, dor de garganta e a presença de massa ou edema cervical.

Este estudo tem por objetivo relatar os achados clínicoradiológicos, a abordagem clínica e/ou cirúrgica, bem como a evolução dos pacientes com diagnóstico de infecção cervical profunda, comparando os achados com dados da literatura.

\section{CASUÍSTICA E MÉTODO}

Foram estudados prospectivamente 57 pacientes internados, no período de janeiro de 1999 a janeiro de 2001, na Divisão de Clínica Otorrinolaringológica do Hospital das Clínicas da Faculdade de Medicina da Universidade de São Paulo com o diagnóstico de infecção dos espaços cervicais profundos (abscesso, celulite ou fasceíte necrotizante). Em todos os casos houve confirmação do diagnóstico através de exame físico, raio-x cervical, ultra-som e/ou tomografia computadorizada. Os espaços cervicais acometidos foram definidos pelos achados de imagem (sobretudo tomografia computadorizada) e/ou cirúrgicos. Raio-x de tórax foi sempre realizado para pesquisar alargamento do mediastino e, em qualquer dúvida, foi realizada tomografia computadorizada de tórax.

Todos os pacientes foram internados, sendo garantida a permeabilidade das vias aéreas e introduzidos antibióticos por via parenteral. A escolha do antibiótico utilizado baseouse no foco primário de infecção e na gravidade do caso.

Corticosteróides foram utilizados por um período médio de 72 horas, desde que não houvesse nenhuma contraindicação como diabetes, disfunção hormonal, hipertensão arterial sistêmica não controlada, doença dispéptica ativa, hipersensibilidade à droga e imunodepressão.

Drenagem cirúrgica, em centro cirúrgico ou prontosocorro, foi prontamente realizada quando requerida pela gravidade do caso ou após exames de imagem que mostrassem coleção. Culturas para aeróbios e anaeróbios foram realizadas quando material purulento era obtido por drenagem espontânea ou durante os procedimentos cirúrgicos.

Foram analisados os seguintes aspectos desta população: idade, sexo, antecedentes pessoais, diagnóstico, etiologia, espaços cervicais acometidos, número de espaços envolvidos concomitantemente, microbiologia das culturas, antibióticos utilizados e evolução dos pacientes.

\section{RESULTADOS}

A nossa casuística é composta por 57 pacientes sendo 32 homens $(56,1 \%)$ e 25 mulheres $(43,9 \%)$. A idade variou de 2 meses a 65 anos, com média de 30,4 \pm 17,2 anos. A distribuição dos pacientes por idade pode ser vista no Gráfico 1. 
Quanto aos antecedentes, observou-se que 2 pacientes $(3,5 \%)$ eram tabagistas, $1(1,7 \%)$ etilista e $5(8,8 \%)$ tabagistas e etilistas. Diabetes foi a doença de base mais

Tabela 2. Distribuição dos espaços cervicais profundos acometidos

\begin{tabular}{lc}
\hline Espaços Comprometidos & $\mathrm{N}(\%)$ \\
\hline Submandibular & $33(57,9 \%)$ \\
Sublingual & $15(26,3 \%)$ \\
Parafaríngeo & $14(24,6 \%)$ \\
Mastigatório & $14(24,6 \%)$ \\
Submentoniano & $8(14 \%)$ \\
Parotídeo & $7(12,3 \%)$ \\
Visceral Anterior & $7(12,3 \%)$ \\
Periamigdaliano & $4(7 \%)$ \\
Bucal & $4(7 \%)$ \\
Retrofaríngeo & $4(7 \%)$ \\
Pré-epiglótico & $2(3,5 \%)$ \\
Infratemporal & $2(3,5 \%)$ \\
Canino & $2(3,5 \%)$ \\
Carotídeo & $2(3,5 \%)$ \\
Mediastino & $1(1,7 \%)$ \\
Perivertebral & $1(1,7 \%)$ \\
\hline
\end{tabular}

Tabela 3. Bactérias isoladas nas culturas de material colhido das infecções

\begin{tabular}{lclr}
\hline Aeróbios & $\mathrm{N}(\%)$ & Anaeróbios & $\mathrm{N}(\%)$ \\
\hline Streptococcus viridans & $22(41,5 \%)$ & Peptostreptococcus micros & $3(5,7 \%)$ \\
Staphilococcus & & & \\
aureus & $11(20,7 \%)$ & Peptostreptococcus sp & $2(3,8 \%)$ \\
Haemophilus influenza & $2(3,8 \%)$ & Peptostreptococcus magnus & $1(1,9 \%)$ \\
Streptococcus pyogenes & $1(1,9 \%)$ & Peptostreptococcus anaerobius 1(1,9\%) \\
Streptococcus epidermidis & $1(1,9 \%)$ & Prevotella oris & $1(1,9 \%)$ \\
Streptococcus b-hemolítico & $1(1,9 \%)$ & Prevotella corporis & $1(1,9 \%)$ \\
Klebsiella pneumoniae & $1(1,9 \%)$ & Prevotella intermadia & $1(1,9 \%)$ \\
Corynebacterium sp & $1(1,9 \%)$ & Prevotella melaninogenica & $1(1,9 \%)$ \\
Neisseriasp & $1(1,9 \%)$ & Clostridium bifermentalis & $1(1,9 \%)$ \\
Acinetobacter baumanii & $1(1,9 \%)$ & Lactobacillus sp & $1(1,9 \%)$ \\
Pseudomonas aeruginosa & $1(1,9 \%)$ & Fusobacterium varium & $1(1,9 \%)$ \\
& & Propiniumbacterium acnes & $11,9 \%)$ \\
\hline Total & $43(81,1 \%)$ & Total & $15(28,3 \%)$ \\
\hline
\end{tabular}

Tabela 4. Antibióticos utilizados

\begin{tabular}{lc}
\hline Antibióticos & $\mathrm{N}(\%)$ \\
\hline Penicilina Cristalina e Metronidazol & $31(54,4 \%)$ \\
Clindamicina & $8(14 \%)$ \\
Penicilina & $3(5,3 \%)$ \\
Clindamicina e Cefalosporina de $3^{\mathrm{A}}$. geração & $2(3,4 \%)$ \\
Penicilina Cristalina e Cloranfenicol & $2(3,4 \%)$ \\
Cefalotina & $2(3,4 \%)$ \\
Oxacilina & $1(1,8 \%)$ \\
Cloranfenicol & $1(1,8 \%)$ \\
Oxacilina e Cloranfenicol & $1(1,8 \%)$ \\
Penicilina Cristalina e Cefalosporina de $3^{\mathrm{A}}$. G & $1(1,8 \%)$ \\
Oxacilina e Metronidazol & $1(1,8 \%)$ \\
Metronidazol e Cefalosporina de 3 ${ }^{\mathrm{A}}$. geração & $1(1,8 \%)$ \\
Clindamicina e Amicacina & $1(1,8 \%)$ \\
Vancomicina e Imipenem & $1(1,8 \%)$ \\
Penicilina Cristalina, Metronidazol e Amicacina & $1(1,8 \%)$ \\
\hline Total & $57(100 \%)$ \\
\hline
\end{tabular}

prevalente ( 7 casos - 12,3\%), também existindo hipertensão, hepatopatia esquistossomótica, epilepsia e usuário de drogas endovenosas ( 1 caso de cada -1,7\%).

A maioria dos casos apresentou abscesso cervical $(87,7 \%), 5$ casos $(8,8 \%)$ apenas celulite e 2 casos $(3,5 \%)$, fasceíte necrotizante.

A distribuição das etiologias relacionadas às infecções cervicais profundas pode ser vista na Tabela 1 .

O comprometimento dos espaços cervicais esta apresentado na Tabela 2, sendo que o acometimento de mais de um espaço ocorreu simultaneamente em 34 casos (59,6\%).

Culturas foram realizadas com material purulento obtido de 53 pacientes (93\%). Destas, 28,3\% não apresentaram crescimento bacteriano. Culturas mistas, contendo mais de um organismo ocorreu em 11 pacientes $(20,8 \%)$.

A Tabela 3 mostra a distribuição dos microorganismos aeróbios e anaeróbios isolados em cultura de material colhido em 53 pacientes.

O tratamento antibiótico utilizado pode ser observado na Tabela 4.

Cinqüenta e quatro pacientes $(94,8 \%)$ melhoraram do quadro, sem complicações. Apenas 2 pacientes $(3,4 \%)$ tiveram deiscência de sutura durante o tratamento e $1(1,7 \%)$ faleceu devido à mediastinite.

\section{DISCUSSÃO}

\section{Apresentação Clínica}

Classicamente, as infecções dos espaços cervicais podem ocorrer em qualquer idade, independente do sexo do paciente. Neste estudo observou-se uma maior incidência em adultos com idade média de 30,4 anos dentro de uma variância de 2 meses a 65 anos, como demonstrado na Gráfico 1. Houve envolvimento de ambos os sexos com discreto predomínio de homens $(56,1 \%)$.

Uma variedade de outras causas tem sido associada às infecções dos espaços cervicais, incluindo sialoadenites, traumas do trato aerodigestivo durante entubação orotraqueal e endoscopia digestiva alta, ingestão de corpos estranhos ${ }^{2}$, traumas penetrantes e/ou contusões cervicais e lesões congênitas cervicais, especialmente os cistos de fendas branquiais, que se associam freqüentemente as infecções cervicais recorrentes ${ }^{3}$.

Observamos que o foco odontogênico foi o principal responsável pelas infecções ( $42,1 \%$ dos casos), seguido dos focos amigdalianos $(17,5 \%)$ e linfadenite cervical abscedada (15,8\%). Esses 3 focos foram responsáveis por 75,4\% da nossa casuística.

Segundo relatos da literatura, estas infecções associamse predominantemente a focos dentários e faríngeos, sendo que a maioria dos pacientes, ao se apresentarem ao serviço médico, referem uma história de infecções de vias aéreas 
superiores, manipulação ou infecção dentária precedendo o quadro. Contudo, alguns pacientes não associam qualquer fator desencadeante.

Dentre as outras causas, observamos ingestão de corpo estranho em $3,5 \%$ dos casos, submandibulite em 1,7\%, cisto de fenda branquial infectado em $1,7 \%$, necrose tumoral $1,7 \%$ e infecção de pele em 5,3\% dos casos, estando de acordo com a literatura ${ }^{4}$. As causas indefinidas ocorreram em somente $8,8 \%$ dos casos, o que está bem abaixo das publicações que a relatam em torno de $25 \%{ }^{5}$ a $37,5 \%{ }^{6}$. Esse fato poderia ser justificado por termos somente incluído no estudo os pacientes que foram internados, pois a principal dificuldade de definir a etiologia geralmente ocorre nas linfoadenopatias, muitas vezes tratadas ambulatorialmente.

As infecções cervicais podem disseminar-se locoregionalmente através de diferentes vias como a hematogênica e a linfática, mas principalmente por extensão direta (contigüidade) dissecando por entre as fáscias, atingindo e envolvendo múltiplos espaços cervicais ${ }^{7}$.

Considerando as relações anatômicas entre os espaços e estruturas da face e a maior incidência de disseminação por extensão direta, é clássico o envolvimento de espaços específicos de acordo com o foco de origem da infecção ${ }^{7}$. As infecções odontogênicas acometem principalmente os espaços submandibular, submentoniano e sublingual e, posteriormente, o mastigatório e o parafaríngeo ${ }^{8}$. Este último, por sua localização na confluência de diversos espaços, também é freqüentemente acometido quando os focos são faríngeos, especialmente por amigdalites ou outras IVAS.

Conforme citado anteriormente, o foco de origem infecciosa mais comum neste estudo foi odontogênico $(42,1 \%)$ seguido das amigdalites $(17,5 \%)$ e linfadenites pós IVAS $(15,8 \%)$. Conforme relatado na literatura, observamos que os espaços mais freqüentemente acometidos foram o submandibular (57,9\%), o sublingual (26,3\%), o parafaríngeo (24,6\%), o mastigatório (24,6\%), o submentoniano (14\%), o periamigdaliano (7\%) e o bucal (7\%).

Estes achados são importantes para nortear a investigação clínico-radiológica dos pacientes, para prever possíveis complicações e planejar a abordagem cirúrgica.

\section{Microbiologia}

Alguns autores chegam a citar que a alta prevalência de casos em que a bacterioscopia direta (Gram) evidencia a presença de bactérias na amostra, porém a cultura é negativa, pode refletir a presença de anaeróbios?.

A microbiologia das infecções dos espaços cervicais caracteriza-se por um predomínio de bactérias Gram positivas, especialmente as espécies de Streptococcus e o Staphylococcus aureus ${ }^{10}$, mas, cada vez mais, a identificação de germes anaeróbios nas culturas enfatiza a importância das infecções polimicrobianas ${ }^{11}$.

Obtivemos material para cultura em 53 casos, destes, $28,3 \%$ não apresentaram crescimento bacteriano. Inúmeras são as causas de ausência de crescimento, como antibioticoterapia prévia, coleta e meio de transporte inadequados e demora no encaminhamento do material para o laboratório. Em caso de bactérias mais sensíveis, a cultura pode ser negativa. As principais bactérias isoladas nas culturas foram os Gram positivos, sendo 41,5\% Streptococcus viridans, 20,7\% Staphylococcus aureus e 5,7\% outras espécies de Streptococcus. Em 20,8\% as culturas foram compostas de flora mista.

Considerando apenas os anaeróbios, as espécies de Peptostreptococcus corresponderam a 13,3\% das culturas e as de Prevotella, 7,6\%, dados estes concordantes com os estudos mais recentes na literatura. Como já enfatizado, são as bactérias com maior dificuldade de isolamento por não resistirem à exposição ao meio ambiente.

A presença de bactérias Gram negativas no estudo, especialmente de $\mathrm{H}$. influenzae $(3,8 \%)$, $\mathrm{K}$. pneumoniae (1,9\%), Corynebacterium sp (1,9\%), Neissseria sp $(1,9 \%)$, Acinetobacter baumanii (1,9\%) e Pseudomonas aeruginosa $(1,9 \%)$ correspondendo a $13,3 \%$ das amostras, aproxima-se de relatos da literatura que chegam a citar 18\% de Gram negativos nas amostras, especialmente o $\mathrm{H}$. influenzae. Este microorganismo pode ser isolado em linfadenite pós-IVAS, mas os demais Gram negativos geralmente são encontrados em flora polimicrobiana ou em pacientes com doenças de base.

A associação de certas doenças sistêmicas às infecções cervicais profundas é freqüentemente citada, sendo o diabetes uma das patologias mais importantes como fator de risco para as infecções complicadas e de evolução letal. Autores como Lee ${ }^{12}$ relatam uma maior susceptibilidade destes pacientes à infecção por bactérias Gram negativas ou espécies de Staphylococccus. Sete pacientes $(12,28 \%)$ neste estudo tinham diagnóstico prévio de diabetes sendo que os organismos isolados na cultura de 6 deles (1 apresentava apenas flegmão), foram: Streptococcus viridans (2 pacientes), S. aureus (1), Klebsiella pneumoniae (1) e flora mista composta de S. viridans, Peptostreptococcus anerobious e $\mathrm{P}$. micros em 2 pacientes. O único paciente com evolução fatal fazia parte deste grupo cuja cultura indicou flora mista.

Os usuários de drogas endovenosas representam um grupo específico que apresenta maior incidência de infecções por Gram positivos especialmente por cepas de Staphylococcus e Streptococcus ${ }^{13}$. Observamos apenas um paciente usuário de drogas e sua cultura revelou S. viridans, H. influezae e Peptostreptococcus.

\section{Abordagem Terapêutica}

Os princípios básicos do tratamento das infecções dos espaços cervicais consistem na manutenção das vias aéreas superiores, na terapia antimicrobiana e na drenagem cirúrgica.

A manutenção das vias aéreas superiores é a prioridade 
do tratamento e, em certas situações como nos abscessos envolvendo diversos espaços cervicais com diminuição ou mesmo oclusão dos espaços aéreos, a entubação orotraqueal, a traqueostomia ou a cricotireoidostomia devem ser consideradas ${ }^{20}$.

A terapia antimicrobiana deve ser introduzida no momento da avaliação clínica do paciente e a nossa escolha baseou-se na cobertura ampla das bactérias predominantemente envolvidas, os Gram positivos e anaeróbios, por via endovenosa. A combinação de Penicilina e Metronidazol foi utilizada em 54,4\% dos casos. O ajuste destas drogas, quando necessário, realizou-se após o resultado das culturas com antibiograma, direcionando o tratamento para uma cobertura específica das bactérias isoladas.

A maioria dos trabalhos na literatura cita o uso de combinações antimicrobianas de amplo espectro para a cobertura de Gram positivos, Gram negativos e anaeróbios como escolha inicial para o tratamento ${ }^{6}$. Entretanto, nossos dados mostram que a cobertura inicial para Gram negativos seria benéfica somente em 12,3\% dos casos, sendo estes isolados geralmente em flora múltipla.

Considerando especificamente as etiologias mais freqüentes como S. aureus, estreptococos hemolíticos e anaeróbios, recomenda-se, na literatura, o uso de Penicilina para os Gram positivos e anaeróbios, Clindamicina ou Metronidazol para anaeróbios e Cefalosporinas de $3^{\text {a }}$ geração ou os aminoglicosídeos para os Gram negativos.

Em nosso estudo a associação de 2 antimicrobianos foi a escolha em $72 \%$ dos pacientes e a combinação mais utilizada foi de Penicilina Cristalina e Metronidazol. Essa combinação pode ser substituída com vantagem pela Clindamicina, que apresenta melhor cobertura para S. aureus, tem boa penetração em osso e pode ser administrado por via oral. Embora seu espectro para anaeróbios seja menor que o do Metronidazol, tem boa cobertura para os anaeróbios envolvidos nas infecções cervicais. Três antibióticos foram utilizados apenas em 1 paciente (1,7\%) e, em 26,2\% dos casos um antibiótico foi utilizado isoladamente.

Opiniões divergentes quanto ao momento adequado da abordagem cirúrgica destas infecções são observadas na literatura. Certos autores ${ }^{15}$ advogam a intervenção cirúrgica precoce mesmo nos casos em que os achados clínicotomográficos são compatíveis apenas com celulite, sem coleção purulenta, porém a maioria ainda sustenta uma conduta mais tradicional, optando pela cirurgia apenas quando há evidências de liquefação em meio aos tecidos ${ }^{16}$. Neste estudo $87,7 \%$ dos pacientes apresentaram achados clínicos e/ou tomográficos de coleção purulenta e 2 pacientes $(3,5 \%)$, de fasceíte necrotizante, sendo abordados cirurgicamente. Apenas em 5 pacientes $(8,8 \%)$ o processo infeccioso foi diagnosticado como celulite, sendo, nestes casos, empregado apenas tratamento clínico. Deve-se atentar, no entanto, que a celulite pode evoluir para abscessos, sendo necessária a drenagem.
A partir desta abordagem, obtivemos resultado satisfatório com cura, sem seqüelas, em 94,8\% dos casos, 2 pacientes apresentaram deiscência da ferida cirúrgica e 1 evoluiu para óbito devido ao quadro de fasceíte necrotizante complicada com mediastinite. Estudos prévios sobre as infecções dos espaços cervicais citam incidências mais altas de complicações, em torno de $19 \%{ }^{17}$, enfatizando os casos de fasceíte necrotizante, cuja taxa de mortalidade varia de $38 \%$ a $50 \%{ }^{18}$, requerendo debridamentos repetidos e antibioticoterapia prolongada.

Outras complicações como pneumonia aspirativa, infarto agudo do miocárdio, cetoacidose diabética, ruptura de artéria carótida, septicemia e falência de múltiplos órgãos também são citadas ${ }^{19,20,21}$. Atenção especial deve ser dada às complicações vertebrais, observadas em 2 casos, principalmente a osteomielite vertebral, a infecção de disco intervertebral (discite) e o abscesso epidural de coluna cervical, geralmente conseqüências da extensão direta da infecção através da fáscia pré-vertebral, pois estes quadros perfazem uma baixa porcentagem das complicações, porém representam alta morbidade para o paciente ${ }^{22,23}$.

\section{CONCLUSÃO}

O tratamento agressivo das infecções cervicais profundas, com a manutenção da via aérea, antibioticoterapia e drenagem cirúrgica precoce reduzem suas complicações. A cobertura para Gram positivo e anaeróbios é suficiente na maioria dos casos, devendo ser ampliada em casos graves ou quando o foco primário justifique a incidência de Gram negativos. Os exames radiológicos auxiliam no diagnóstico diferencial entre celulite e abscesso e orientam o tratamento.

\section{REFERÊNCIAS BIBLIOGRÁFICAS}

1. Tschiassny K. Ludwig's Angina - A surgical approach based on anatomical and pathological criteria. Ann Otol Rhinol Laryngol 1947;56:937-945.

2. Tsai YS, Lui CC. Retropharyngeal and epidural abscess from swallowed fish bone. Am J of Emerg Med 1997;15(4):381-382.

3. Nusbaum AO, Som PM, Rothschild MA, Shugar JMA. Recurrence of a deep neck infection - A clinical indication of an underlying congenital lesion. Arch Otol Head Neck Surg 1999;125(12):1379-1382.

4. Szuhay G, Tewfik TL. Peritonsillar abscess or cellulites? A clinical comparative pediatric study. The Journal of Otolaryngology 1998;27(4):206-212.

5. Pontell J, Har-El G, Lucente FE. Retropharyngeal abscesses: Clinical review. ENT 1995;74(10):701-704

6. El-Sayed Y, Al Dousary S. Deep-neck space abscesses. The Journal of Otolaryngology 1996;25(4):227-233.

7. Lazor JB, Cunningham MJ, Eavey RD, Weber AL. Comparison of computed tomography and surgical findings in deep neck infections. Otol-Head and Neck Surgery 1994;111(6):746-750.

8. Kim HJ, Park ED, Kim JH, Hwang EG, Chung SH. Odontogenic versus nonodontogenic deep neck space infections: CT manifestations. Journal of Computer Assisted Tomography 1997; 21(2):202-208 
9. Gidley PW, Ghorayeb BY, Stiernberg CM. Contemporary management of deep neck space infections. Otol-Head and Neck Surgery 1997;116(1):16-22.

10. Nagy M, Pizzuto M, Backstrom J, Brodsky L. Deep neck infections in children: A new approach to diagnosis and treatment. Laryngoscope 1997;107:1627-1634.

11. Gianoli GJ, Espinola TE, Guarisco JL, Miller RH. Retropharyngeal space infection: changing trends. Otol-Head and Neck Surgery 1991;105(1):92-100.

12. Lee KC, Tami TA, Echaves M, et al. Deep neck infections in patients at risk for acquired immunodeficiency syndrome. Laryngoscope 1990;100:915-919.

13. Tom MD, Rice DH. Presentation and management of neck abscess: a retrospective analysis. Laryngoscope 1988;98:877-881.

14. Nagy M, Bacstrom J. Comparison of the sensitivity of lateral neck radiographs and computed tomography scanning in paediatric deepneck infections. Laryngoscope 1999;109:775-779.

15. Flynn TR. Odontogenic infections. Oral maxillofac Surg Clin North Am 1991;3:311-329.

16. Endicott JN, Nelson RJ, Saraceno CA. Diagnosis and management decisions in infections of deep fascial spaces of the head and neck utilizing computerized tomography. Laryngoscope 1982;92:630633.

17. Sethi DS, Stanley RE. Deep neck abscess - changing trends. Journal Laryngol Otol 1994;108:138-143.

18. Miller WD, Furst IM, Sandor GKB, Keller MA. A prospective, blinded comparison of clinical examination and computed tomography in deep neck infections. Laryngoscope 1999;109:18731879 .
19. Kaplow HJ, Eichel BS. Deep neck infections. Otolaryngology 1980;3:14-17.

20. Langerbrunner DJ, Dajani S. Pharyngomaxillary space abscess with carotid artery erosion. Arch Otol 1971;94:447-452.

21. Levitt GW. The surgical treatment of deep neck infections. Laryngoscope 1971;81:403-411.

22. Piccolo R, Passanisi M, Chiaramonte I, et al. Cervical spinal epidural abscesses - A report on five cases. Journal of neurosurgical Sciences 1999;43(1):63-67.

23. Saban KL, Ramsis FG. Spinal cervical infection: A case report and current update. Journal of Neuroscience Nursing 1998;30(2):105115.

24. Becker M, Zbären $P$, Hermans R et al. Necrotizing fasciitis of the head and neck: Role of CT in diagnosis and management. Radiology 1997;202:471-476.

25. Brook I. Microbiology of abscesses of the head and neck in children. Ann Otol Rhinol Laryngol 1987;96:429-433.

26. Brook I. Diagnosis and management of anaerobic infections of the head and neck. Ann Otol Rhinol Laryngol 1992;101:9-15.

27. Choi SS, Vezina G, Grundfast KM. Relative incidence and alternative approaches for surgical drainage of different types of deep neck abscesses in children. Arch Otol Head Neck Surg 1997; 123(12):1271-1275.

28. Delap TG, Grant WE, Dick R, Quiney RE. Retropharyngeal abscess - an unusual complication of anorexia nervosa. Journ Laryngol Otol 1996;110:483-484.

29. Krespi,YP, Lawson W, Blaugrund SM. Massive necrotizing infections of the neck. Head Neck Surg 1981;3:474-481.

30. Nicklaus PJ, Kelley PE. Management of deep neck infection. Pediatric Clinics of North America 1996;43(6):1277-1297. 